Caesarean section is but rarely indicated, and should be reserved for special cases, as follows: (1) When the patient is a primigravida with a rigid undilated os and central placenta praevia, and the foetus is known to be alive. (2) When the patient is a multipara who has lost a lot of blood and can ill afford to lose any more. If I might emphasize one point in the treatment of ante-partum haemorrhage it is that the condition is one in which the advantages of treatment in a hospital or nursing home are very great.

Version.

I have already mentioned the value of external version during the later weeks of pregnancy to convert a breech presentation into a vertex. In such cases it may be easy, but an anaesthetic is often required. Version is also the correct treatment for a transverse lie, and in certain cases of placenta praevia. Version should never be performed to effect delivery when the presentation is a vertex and forceps have failed to effect delivery, for in such a case there is great danger of rupturing the uterus. This danger is very real in a transverse lie if version is attempted late in labour. Whenever the uterus is becoming irritable, and the pains are strong and frequent and cannot be completely abolished by anaesthesia, it is unsafe to attempt version. In such a case the foetus is nearly always dead, and its life need not be considered as in any way comparable with the mother's safety. Delivery should therefore be effected after perforation of the foetal head in a vertex presentation, or after decapitation for a transverse lie.

In placenta praevia and in accidental haemorrhage the uterine wall may be weak in places and easily ruptured if any force is used in performing version. Version should not be persevered with if reasonable attempts are unsuccessful, as the failure may be due to insufficient liquor amnii.

Post-partum Haemorrhage.

As in ante-partum haemorrhage, the cause of postpartum haemorrhage is generally (1) uterine exhaustion or lack of tone; (2) some placenta or membranes left behind in the uterus. In my opinion a most important predisposing cause of these two conditions is chloroform when given in fair quantity for a prolonged period, especially if pushed to a sufficient extent to allow the application of instruments. There is nothing which abolishes uterine contractions so quickly and effectually as chloroform, or, to a lesser extent, ether; and if the anaesthetic is given for any length of time there is likely to be difficulty in the third stage owing to deficient uterine contractions and post-partum haemorrhage will occur.

Post-partum haemorrhage is also likely to follow the overdistension of the uterus by twin pregnancy or hydramnios. In such cases, therefore, it is very important to keep very careful watch over the uterus for at least an hour after the delivery of the infant, and to have preparations in hand for a hot vaginal and intrauterine douche. If haemorrhage occurs after the expulsion of the placenta a hypodermic injection of pituitary extract should be given at once, and should be followed by a hypodermic injection of ergot.

\section{Caesarean Section.}

The comparative ease and safety with which Caesarean section can be performed has led to a considerable increase in the number of the operations. The scope of the opera. tion has been widened to include not only the extreme cases of contracted pelvis, but also cases of pelvic contraction of a minor degree, of malpresentations, of eclampsia, of placenta praevia, and of accidental haemorrhage. The increased frequency with which the operation has been performed during the last few years has been accompanied by, first, an increased maternal mortality, and secondly, a considerable number of unfavourable postoperative sequelae of which the most serious is rupture of the uterine scar in a subsequent pregnancy. The result is that the pendulum has begun to swing in the opposite direction, and the number of cases of Caesarean section, in the London hospitals at any rate, has dropped considerably. The indications for Caesarean section should certainly be as definite as for any other major operation. Before Caesarean section is undertaken the following points should be ascertained: (1) that the foetus is alive; (2) that the foetus has no reasonable chance of delivery per vias naturales; (3) that the labour is not far advanced; (4) that there is no undue risk to the mother. The great risk in the operation is sepsis from the uterus, resulting in death of the mother from peritonitis or septicaemia, or breaking down of the abdominal or uterine wound. If any sepsis be present the uterine wound will not heal well so as to leave a good scar. Weakness of the scar may also result from inefficient suture of the uterine wound. There is no operation in which it is more important to have favourable conditions for the carrying out of a perfect aseptio technique. The risk of a weak scar has of recent years been overcome by making the incision into the uterus through the lower uterine segment after pushing down the bladder. The objection to this is that the operation is more difficult, and if the child is large it may be difficult to extract. The incision has much to recommend it, and I consider it a marked advance on the old incision. Caesarean section in cases of eclampsia has a high mortality, among hospital patients at any rate, and is performed less frequently than formerly. Caesarean section in cases of central placenta praevia or severe accidental haemorrhage is, in my opinion, a good method of treatment in picked cases.

\section{Unreduced Occipito-posterior Position.}

The difficulties likely to arise in connexion with an occipito-posterior position result from uterine inertia, prolonged labour, and the application of forceps. Following these there may be a torn perineum, torn cervix, or injury to the bladder, and permanent weakness of the pelvic floor resulting from difficult forceps delivery. There is also a possibility of rupture of the uterus during forceps or manipulations. The correct treatment consists in making sure of the diagnosis when the condition is suspected, and promoting flexion of the foetal head during the first stage, by posture. If the occiput does not rotate after a reasonable time in the second stage an anaesthetic should be given and the head, with the body of the foetus, should be rotated. If reduction cannot be performed and the head is well down, tentative and judicious trial of axis traction forceps may be made. If the head does not descend with gentle traction, perforation of the foetal head should be performed.

After the paper there was a discussion, and questions were asked by many members present. In reply Mr. Luker pointed out that he did not think that any harm resulted from giving drops of chloroform during the latter part of the second stage, but he wished to emphasize the danger, in his opinion, of large quantities for any length of time.

\section{ACUTE LUMBAGO TREATED BY THE INJECTION OF QUININE AND UREA.}

BY

H. S. SOUTTAR, M.Ch.Oxon., F.R.C.S.Eng.,

SURGEON (WITH CHARGE OF OUT-PATIENTS), LONDON HOSPITAL.

OF common minor ailments there is none which may be more completely paralysing than acute lumbago. In an instant an individual in the full vigour of health may find himself unable to make the slightest movement of the trunk without the most exquisite pain, and he may remain confined to bed for weeks as an absolute cripple. Even in the slightest cases, though the acute symptoms may pass off in a few days, it will be ten days at least before he can stoop with freedom and comfort. In severe cases the slightest movement, or even a sudden noise, may induce a spasm of pain, with a violent contraction of the lumbar muscles, a reflex attempt at protective fixation. The contraction may last for several seconds, and only slowly and cautiously will the patient allow the muscles to relax.

An attack is often brought on by a chill following exertion, such as may occur from sitting in clothes damp from perspiration: An attempted movement produces a spasm 
of pain, and after that free movement is impossible. The pathological basis is very obscure and probably involves a number of different factors. The circulation in the muscles, diminished by cold, is unable to remove the waste products resulting from recent activity. Probably the muscle responds to stimuli by an irregular spasm, and a few of its fibres are torn from their origin. This conception would $i t$ least explain the fact that it is almost always at a point of attachment that tenderness is most marked.

Though the sudden onset is strongly suggestive of a traumatic origin there is usually a slight but definite constitutional disturbance, and it seems probable that the trauma would be ineffective in the absence of some error in general metabolism, perhaps due to a low grade of infection. But the local symptoms are so severe as entirely to absorb the patient's attention. A close examination will indeed show that the source of these symptoms is even more narrowly localized than the patient imagines, and that the pain spreads from a very small focus characterized by exquisite tenderness to deep pressure. This focus may be situated anywhere in the lumbar muscles, and in addition to its excessive sensitiveness may be marked by a slight local swelling, with an oedema which pits on pressure.

The precise localization of the focus of pain naturally suggests some local form of treatment, and a large number of methods have been recommended. Radiant heat, dry cupping, heavy massage, the insertion of long needles, and the actual cautery, all have their advocates, but the more lenient methods are tedious, whilst the more drastic are somewhat oriental. It occurred to me that the injection of a local anaesthetic would be a rational method to employ and could at least do no harm. I was scarcely prepared, however, for the instantaneous cures which I have observed, and still less for their permanence.

The anaesthetic I chose was quinine and urea hydrochloride in 1 per cent. solution, and I selected it because of the length of time, up to several days, for which the anaesthesia may last. I thought at first that the action might be purely mechanical and I tried normal saline solution, but the effect proved to be only transitory. $5 \mathrm{c.cm}$. of the solution is injected through a long fine needle into the centre of the tender spot; within ten minutes the acute pain has entirely disappeared and the patient can move freely; a sense of stiffness remains, and a curious feature is that the patient may complain of a general malaise. It has seemed to me probable that some constitutional disturbance is a regular feature of the condition, but that it is masked by the severity of the local pain.

The following cases illustrate clearly the method employed and the results.

A lady, aged 45, was suddenly seized with severe pain in the right loin. The pain came in spasms so severe that whilst they lasted the right leg appeared to be paralysed and the possibility of a lesion of the lumbar roots was even considered. She lay in bed unable to move, the slightest attempt at movement provoking an attack of pain. Massage, radiant heat, and moist heat only gave partial and temporary relief. Below the iliac crest and just external to the right sacro-iliac joint was an area about the size of a florin, slightly boggy to pressure, and exquisitely tender. Into this area a hypodermic needle was passed till it reached the bone, and $5 \mathrm{c.cm}$. of a 1 per cent. solution of quinine and urea hydrochloride was injected. Ten minutes later the patient found that she could move without producing a spasm and in two hour she could get up and walk. There were no further two hour beyond a feeling of stiffness which lasted for ten days she declared herself cured.

A lady, aged 52, passing through London was seized with violent pain in the lower right lumbar region on attempting to rise from bed in the morning. She had had a previous attack of lumbago which confined her to bed for three weeks (under the care of an osteopath who informed her that she had dislocated her sacro-iliac joint!). There was a tender spot just external to the right sacro-iliac joint, and as the case was one of moderate severity I tried the injection of $5 \mathrm{c.cm}$. of normal saline solution. Marked relief followed, but only lasted a few hours. An injection of quinine was, however, followed by complete relief. An injection of quinine was, however, followed by complete relief, and two days
later she was able to travel. A month later she wrote to tell that she had had no further pain and was perfectly well.
the

It seems to me that a method at once so simple and so safe ought to be generally known and that it must have extensive applications. I have little doubt that it would be equally effective in those similar cases of torticollis an.l pleurodynia where a localized tender spot is always a marked feature.

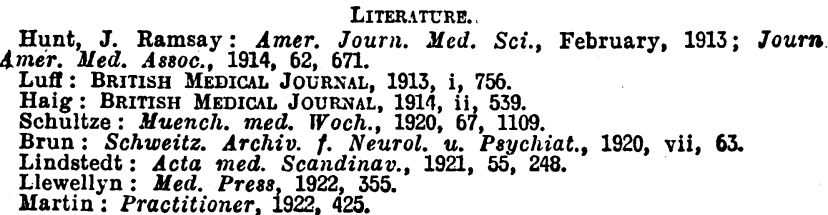



\section{THE EFFECT OF PARATHYROID ON THE BLOOD SUGAR CURVE AFTER INSULIN.}

BY

\author{
W. DEVEREUX FORREST, M.D., \\ HONORARY MEDICAL REGISTRAR, ROYAL VICTORIA INFIRMARY, \\ NEWCASTLE-ON-TYNE.
}

Wister and Smith have recently reported ${ }^{1}$ that when insulin and parathyroid are injected simultaneously into rabbits, convulsions (due to reduction of the amount of sugar in the blood) ensue in a much shorter period and with a much smaller dose of insulin (one-third to one-fourth of the normal dose). I applied these results to the human subject, and found that parathyroid was almost, if not quite, as efficacious when given by mouth, and in the cases reported below this method was used. The procedure in all the cases was the same. The patient was not allowed any food beyond clear soup for five hours before injection. The parathyroid and insulin were administered, and the first blood taken within five minutes, and blood-sugar estimations were carried out hourly from that time to the end of the observation period. No food was given until after the last estimation.

\section{CASE I.}

H. S., male, aged 36. Diabetes diagnosed two years ago. He was treated on a modified Allen diet, but was careless, and persistently overstepped it by small amounts. He had got worse of late, and developed marked ketonuria in July, without any alteration of diet. Twenty units of A.B. insulin given aloue. Blood sugar: 0.310 $0.265,0.227,0.162,0.155,0.187$, and 0.234 per cent.

Twenty units of A.B. insulin with four $1 / 10$ grain parathyroid tablets. Blood sugar : $0.329,0.257,0.196,0.141,0.118,0.102,0.136$, and 0.169 per cent.

\section{CASE Ir.}

J. A., male, aged 45. First symptoms in February, 1922. Was thirsty, and lost over 2 st. in weight. Was treated on old lines thirsty, and lost over 2 st. in weight. Improved fcr a few months, with codeine, and restriction of bread. Improved fcr a few months, but lost ground again towards the end of the year. Admitted to the infirmary in January, and was given three doses of insulin, and put on to the Allen dietary. Was a bad patient and did not adhere
to his diet. Returned again in September, and was treated with nsulin.

Twenty units of insulin given alone. Blood sugar: $0.342,0.292$, $0.256,0.201,0.216,0.238$, and 0.277 per cent.

Twenty units of insulin with twelve $1 / 10$ grain tablets of parathyroid. Blood sugar: $0.329,0.236,0.195,0.149,0.125,0.094$, and 0.081 per cent.

H. R., female, aged 55 . Chronic case. Had had glycosuria for H. R., female, aged 55. Chronic case. Had had glycosuria for four and a half years to her knowledge. Had been thirsty occa. sionally, but main complaint was pruritus. Present weight 13

had been 17 st. The blood sugar curve was definitely diabetic.
Twenty units insulin given alone. Blood sugar : 0.362 per cent., Twenty units insulin given alone. Blood s

missing, $0.214,0.156,0.172$, and 0.224 per cent.
Twenty units insulin with ten parathyroid tablets. Blood sugar : $0.339,0.246,0.182,0.125,0.107,0.091,0.088$, and 0.122 per cent.

\section{CASE IV.}

L. S., male, aged 23. In January this year he showed typical symptoms. He was treated by a modification of the Allen diet, and improved greatly. In July he felt worse again, and it was found that his tolerance had dropped from 95 grams carbohydrate to 60 . He was readmitted, and given insulin, with marked benefit to and sligessary expense, he dropped the insulin, and became worse again.

Fifteen units insulin given alone. Blood sugar : 0.284, 0.243, 0.216, $0.153,0.167$, and 0.202 per cent.

Fifteen units insulin with six parathyroid tablets. Blood sugar : $0.322,0.246,0.191,0.153,0.124,0.104,0.118$, and 0.143 per cent.

\section{Case v.}

M. M. W., female, aged 26. Diabetes was diagnosed at Christmas, 1922, but she had symptoms for a considerable time previously.
Refused to come into hospital until quite recently, and had had practically no treatment hitherto.

Fifteen units insulin given alone. Blood sugar : $0.410,0.326,0.229$, $0.277,0.229,0.242$, and 0.283 per cent. 